\title{
La vitamina E y la selegilina son efectivos para disminuir la progresión de la enfermedad de Alzheimer
}

Sano MS, Ernesto C, Thomas RG. A controlled trial of selegiline, Alpha-Tocopherol, or both as treatment for Alzheimer disease. New Engl J Med 1997;336:1216-22

\section{Objetivo}

Evaluar la eficacia de la selegina y la Vitamina E, solas o en forma combinada para disminuir la progresión de la enfermedad de Alzheimer.

\section{Diseño}

Ensayo controlado, doble ciego, randomizado, y multicéntrico. Seguimiento a 2 años.

Lugar

23 centros de EE.UU.

\section{Pacientes}

341 pacientes ( $65 \%$ mujeres) con una edad media de 73.5 años y una duración promedio de la enfermedad de 5 años. El puntaje basal promedio del Minimental Test de Folstein fue de 13 y el puntaje clínico de demencia de 11. Se excluyeron los pacientes con otra enfermedad neurológica, los que estuvieran recibiendo psicofármacos y los institucionalizados.

\section{Intervención}

Se administró selegilina $10 \mathrm{mg} /$ día, Vitamina E 2.000 UI/día, la combinación de ambas o placebo por el término de 2 años.

\begin{abstract}
Medición de Resultados Principales
Tiempo de aparición de alguno de los siguientes eventos: muerte, internación en un geriátrico, pérdida de la capacidad de realizar actividades de la vida diaria o aparición de demencia severa (puntaje clínico de demencia de 3). Para controlar la adherencia al tratamiento se dosaron los niveles de drogas en sangre.
\end{abstract}

\section{Resultados}

A pesar de ser un estudio randomizado, hubo diferencias en los puntajes de Minimental iniciales entre los grupos (el grupo placebo tenía menos severidad de demencia que los otros tres). En el análisis inicial (sin tener en cuenta el puntaje inicial del Minimental) no hubo diferencias estadísticamente significativas entre los 4 grupos con respecto a los resultados. Al tener en cuenta las diferencias basales en el Minimental, se demuestra una diferencia significativa entre la administración de cualquiera de los tratamientos versus el placebo con respecto al tiempo de aparición de alguno de los eventos descriptos: a) selegilina: mediana de tiempo 655 días; $\mathrm{p}=0.012$; b) Vitamina $\mathrm{E}$ : 670 días; $\mathrm{p}=0.001$; c) combinado 585 días; $p=0.049$; d) placebo: 440 días (grupo control).

\section{Conclusiones}

En pacientes con un deterioro moderado por enfermedad de Alzheimer, la administración de Vitamina E o selegilina enlentece la progresión de la enfermedad.

Apoyo económico: National Institute of Health. EE.UU, y entrega de fármacos por parte de los laboratorios que manufacturan las drogas.

\section{Comentario}

El progresivo envejecimiento de la población y el consecuente aumento de la prevalencia de la enfermedad de Alzheimer, ha generado una gran presión en la industria farmacéutica para investigar drogas que sean efectivas contra este mal. Sólo la tacrina y el donepezil han sido aprobados en países desarrollados, ofreciendo una utilidad limitada a un alto costo. Los resultados aqui evaluados son de carácter clínico, lo cual se opone a los utilizados en estudios previos (test de memoria y otras funciones cognitivas) y dificulta la comparación. La randomización intenta evitar la desigual distribución de variables iniciales de los pacientes. La diferencia inicial en el puntaje del Minimental del grupo placebo comparado con el resto, abre un margen dè duda sobre la calidad de la randomización. Esta diferencia fue menor a dos puntos, pero el tenerla en cuenta en el análisis modificó los resultados del estudio. Llamativamente, los pacientes que recibieron selegilina sóla o combinada tenían más prevalencia de signos extrapiramidales (diferencia no significativa) y esto no fue tenido en cuenta en el análisis, Esto podría mostrar a la selegilina como efectiva para la demencia cuando en realidad es efectiva para el extrapiramidalismo. Otro hallazgo a comentar es que teniendo las drogas efecto beneficioso independiente para retardar la enfermedad, no exista efecto aditivo al utilizarlas en forma combinada. Otro problema del presente estudio es que al haber cuatro ramas y muchos resultados a comparar entre ellas, aumenta la probabilidad de encontrar diferencias cuando en realidad éstas no existan. Los ensayos sobre tratamiento que buscan disminuir la progresión de una enfermedad, son difíciles de interpretar y más allá de que demuestren diferencias, son útiles para señalar el camino para futuras investigaciones. En conclusión, a pesar de las dificultades metodológicas, tanto la vitamina E como la selegilina parecen retardar la progresión de la enfermedad de Alzheimer de manera estadísticamente significativa pero queda por determinar la importancia clínica y el comportamiento a más largo plazo de estas diferencias. Mientras tanto los médicos que atendemos a pacientes con demencia podríamos utilizar selegilina o vitamina $\mathrm{E}$, recordando que existe cierta evidencia que la selegilina puede aumentar la mortalidad en pacientes con otra patología como el Parkinson

\section{Referencia}

Treatment for Alzheimer's disease- Searching for breakthrough, Settling for less. New Engl J Med 1997;336:145-7

Dr. Ezequiel García Elorrio Clínica Médica CEMIC 